\title{
„Züri wie neu“: Public Value von Online-Partizipation
}

\author{
Gabriel Abu-Tayeh • Edy Portmann · Matthias Stürmer
}

Eingegangen: 17. Februar 2017 / Angenommen: 12. April 2017 / Online publiziert: 25. April 2017 (C) Der/die Autor(en) 2017. Dieser Artikel ist eine Open-Access-Publikation.

Zusammenfassung Der Public Value berücksichtigt neben den herkömmlichen Zielsetzungen Effizienz und Effektivität auch soziale Faktoren aus der Sicht der Bevölkerung. Bisher haben jedoch nur wenige Studien gemessen, wie die Bevölkerung den Wert von Dienstleistungen der öffentlichen Verwaltung wahrnimmt; in der Regel wird der potentielle Nutzen solcher Dienstleistungen nur im Voraus prognostiziert, da der Wert auf Grundlage von Expertenmeinungen bemessen wird. Diese Forschungslücke schließen wir, indem wir mittels Fragebögen $(n=738$ Teilnehmer) exemplarisch den wahrgenommenen Nutzen der Open-Government-Initiative ,Züri wie neu“ für die Bevölkerung evaluieren. Über diese Plattform können BürgerInnen der Stadt Zürich mit einer einfach zu bedienenden Web- bzw. mobilen Anwendung Schäden und Mängel bei der Infrastruktur melden.

Wie die Auswertung zeigt, bewerten die Teilnehmenden bei der Interaktion mit dem Service besonders den Zeitgewinn (d. h. Effizienz), den Komfort und die Kommunikation (beides Effektivität) positiv. Überdurchschnittlich hohe Werte zeigen sich aber auch bei der Bildung von Vertrauen in die Verwaltung, der wahrgenommenen eigenen Informiertheit und dem Gefühl partizipativ in Entscheidungsfindungen (alles soziale Werte) beteiligt zu sein. Dies zeigt, dass Faktoren für Effizienz und Effektivität aus Sicht der Bevölkerung nicht das gesamte Wertespektrum von

\footnotetext{
G. Abu-Tayeh $(\bowtie)$

Institut für Wirtschaftsinformatik, Technologiezentrum Wirtschaftsinformatik, Universität Bern, Engehaldenstraße 8, 3012 Bern, Schweiz

E-Mail: gabriel.abu-tayeh@iwi.unibe.ch

E. Portmann $(\bowtie)$

Institut für Wirtschaftsinformatik, Universität Bern, Engehaldenstraße 8, 3012 Bern, Schweiz

E-Mail: edy.portmann@iwi.unibe.ch

M. Stürmer $(\bowtie)$

Institut für Wirtschaftsinformatik, Forschungsstelle Digitale Nachhaltigkeit, Universität Bern,

Engehaldenstraße 8, 3012 Bern, Schweiz

E-Mail: matthias.stuermer@iwi.unibe.ch
} 
Dienstleistungen der öffentlichen Verwaltung abbilden können sondern auch soziale Vorteile relevant sind.

Schlüsselwörter Public Value · Online Partizipation · Open Government • Vertrauen · Zueri wie neu

\title{
„Züri wie neu“: Public Value of Online-Participation
}

\begin{abstract}
Next to the common goals efficiency and effectiveness the notion of public value considers also social aspects relevant to society. However, only a few studies have measured how the population appreciates the value of services by the public sector. So far the potential use of such services has only been predicted in advance based on expert opinion. We close this research gap by an empirical study $(n=738)$ on the open government platform "Züri wie neu", a Swiss adoption of the FixMyStreet initiative from UK. On this platform citizens of Zürich report damages and defects of infrastructure with a user-friendly web and mobile application.

The results indicate that the participants appreciate time-savings (efficiency), comfort, and interaction with the public service (both effectiveness). In addition we observed above average trust-building with the government, personal knowledgeability and the feeling of participatory decision making (all three of them social values). This explains why efficiency and effectiveness are not the only benefits for the citizens by such platforms but social values are relevant for them, too.
\end{abstract}

Keywords Public Value · Online Participation · Open Government · Trust · Zueri wie neu

\section{Von Kosteneffizienz zum Gemeinwohl}

Ansätze aus dem Public Management, die den Erfolg von Dienstleistungen der öffentlichen Verwaltung (bspw. Online-Partizipation) messen, konzentrieren sich stark auf Messgrößen für Kosteneffizienz. Um dieser Eindimensionalität entgegenzuwirken, wurden in Ansätzen des New Public Management auch Faktoren für Effektivität einbezogen, um öffentliche Organisationen in ähnlicher Weise führen zu können wie private Organisationen (Bryson et al. 2014). Doch auch Messgrößen für Effizienz und Effektivität zusammen können nicht das breite Spektrum sozialer Werte erfassen, die die Bevölkerung von der öffentlichen Verwaltung erwartet und dort im besten Fall auch vorfindet (Meynhardt 2009). Nicht minder wichtig für die Evaluation von Dienstleistungen im öffentlichen Sektor sind etwa die Vertrauenswürdigkeit der öffentlichen Verwaltung in den Augen der BürgerInnen oder die Herstellung sozialer Gleichheit. Darum folgen wir in unserer Forschung zu Online-Partizipation im öffentlichen Sektor dem Public-Value-Ansatz, der ursprünglich von dem Harvard-Verwaltungswissenschaftler Mark Moore entwickelt wurde und auf eine ganzheitlichere Analyse des Gemeinwohls abzielt (Moore 1995).

Wir untersuchen in diesem Artikel die Frage, ob Online-Partizipation im öffentlichen Sektor von der Bevölkerung als wertvoll empfunden wird. Dies tun wir ex- 
emplarisch anhand des Open-Government-Portals „Züri wie neu“. Dabei handelt es sich um eine Software-Anwendung der Stadt Zürich, mit der die Bevölkerung Schäden an der Infrastruktur direkt der Stadtverwaltung melden kann und diese dazu umgehend Stellung nimmt. Via Webanwendung oder mobile App können der Verwaltung beispielsweise Schlaglöcher in den Straßen, defekte Ampeln oder verschmutzte Parkbänke gemeldet werden (Habenstein et al. 2016). Anhand von Fragebögen (Rücklauf von 738 vollständig ausgefüllten Fragebögen) untersuchen wir empirisch, wie die Bevölkerung den Nutzen dieses Portals wahrnimmt.

Der Beitrag ist folgendermassen aufgebaut: Wir beginnen mit der Erläuterung der theoretischen Hintergründe zu Open Government (Teil 2), Partizipation im öffentlichen Sektor (Teil 3) und den Zusammenhang zwischen Effektivität, Effizienz und Public Value (Teil 4). Dann stellen wir unser Fallbeispiel ,Züri wie neu“ vor (Teil 5). Anschließend beschreiben wir die Datenerhebung und präsentieren die Resultate unserer Umfrage (Teil 6). Der letzte Abschnitt behandelt die Diskussion und die Implikationen unserer Studie für andere Aktivitäten im Bereich von OnlinePartizipation und Open Government sowie ihre Limitationen (Teil 7).

\section{Von Max Weber zu Barack Obama}

Fast ein Jahrhundert lang wurde Verwaltung mit dem Bürokratiemodell von Max Weber dargestellt. Kennzeichnend für dieses Modell ist eine starke hierarchische Struktur, in der die Bürgerinnen und Bürger dem Staat untergeordnet sind. Im Vordergrund stehen strikte Regulierungen und vordefinierte Prozessabläufe, die den Staat vor Willkür, Ungleichbehandlung und Korruption schützen (Weber 1922).

Kritisiert wurde an diesem Bürokratiemodell unter anderem, dass es die Komplexität und die Dynamiken der Umwelt nicht genügend berücksichtigen könne (WalterBusch 1996). Dieser Kritik zufolge war das Recht - als primäres Steuerungsinstrument des Bürokratiemodells - nicht in der Lage, alle denkbaren Umweltzustände durch das Gesetz abzudecken und zu regulieren (Hilgers 2012).

Die komplexe Umweltperspektive erforderte eine stärkere Arbeitsteilung sowie eine Zusammenarbeit zwischen den verschiedenen Abteilungen der Verwaltung und mit den externen Akteuren. Dies führte dazu, dass sich administrativ wahrgenommene öffentliche Aufgabenfelder der Verwaltung organisatorisch mit der Zeit verselbstständigten, privatisiert wurden und sich stärker am Markt orientierten. So wurde das klassische Bürokratiemodell in der öffentlichen Verwaltung vom New Public Management (NPM) abgelöst (Dunleavy und Hood 1994).

Das New-Public-Management-Modell unterscheidet sich vom Bürokratiemodell vor allem darin, dass sich die Orientierung vom Input- auf den Output verlagert hat (Hilgers, 2012): Die BürgerInnen werden nicht mehr als passive Untergebene gesehen, sondern als Kunden öffentlicher Leistungen (Osborne und Gaebler 1992). Entsprechend versteht die öffentliche Verwaltung sich selbst weniger als bürokratische Organisation denn als Anbieter von Dienstleistungen, der die Bedürfnisse der Bevölkerung befriedigt (Denhardt und Denhardt 2000).

Diese verstärkte Kundenorientierung und die Weiterentwicklung der Informations- und Kommunikationstechnologien führen nun dazu, dass sich die Verwaltung 
noch weiter öffnet (Hilgers 2012). Die neuen technologischen Mittel und Interaktionsformen bieten neue Möglichkeiten, die Bevölkerung in Verwaltungsprozesse zu integrieren und diese Prozesse partizipativer zu gestalten. Gemäß Habenstein et al. (2016) bildet dieses moderne Staatsverständnis das Fundament für die Entstehung eines Open Government, für das Barack Obama als Weltbotschafter steht (Obama 2009).

In seinem Memorandum „Transparency and Open Government“ warb der damals neu gewählte vorige Präsident der Vereinigten Staaten Barack Obama für eine Öffnung von Regierung und Verwaltung ${ }^{1}$. Als zentrale Elemente von Open Government benennt er Transparenz, Partizipation und Zusammenarbeit. Er sieht darin eine Möglichkeit, das öffentliche Vertrauen in den Staat und die Zusammenarbeit mit dem Volk zu stärken. Ziel dieser neuen Regierungsform ist, die Demokratie zu fördern und die Regierung auf ein neues Level zu heben und zugleich effizienter zu machen.

\section{Gründe für Partizipation im öffentlichen Sektor}

Die angestrebten Formen von Wissenstransfer, Innovation und Kooperation werden durch das Internet und die sozialen Medien, durch Netzwerkplattformen und andere Portale ermöglicht (Collins 2009). Der Staat übernimmt in der Umsetzung von Open Government zwei verschiedene Rollen: Zum einen bietet er Dienstleistungen für seine Bürger an; zum anderen ist er dafür verantwortlich, dass alle Akteure, die in den politischen Prozess zu integrieren sind, auch tatsächlich zur Teilnahme ermutigt werden (Hilgers 2012).

Unabhängig von den verwendeten Mitteln gibt es nach Fiorino (1990) drei zentrale Argumente dafür, die Bevölkerung im öffentlichen Sektor stärker partizipativ einzubinden:

- ein normatives,

- ein substantielles und

- ein instrumentelles Argument.

Die normative Begründung bezieht sich auf das demokratische Ideal, dass die BürgerInnen die besten Experten sind in Entscheidungsfindungen, weil dies ihr eigenes Leben betrifft. Die substantielle Begründung leitet sich daraus ab, dass Laienurteile über Risiken ebenso gut sein können wie Expertenbeurteilungen. Laienurteile haben gegenüber systematischen Expertenurteilen vor allem dann einen Vorteil, wenn sie kontextspezifisch sind und die Wirkungen der Entscheide von den Laien getragen werden müssen. Die instrumentelle Begründung für den Einbezug der Betroffenen ergibt sich aus der grundlegenden Argumentation von Public Value: Danach erhöht Partizipation die Legitimität politischer Entscheidungen und dadurch auch das Vertrauen in die Entscheidungsträger.

Eine Herausforderung dabei ist jedoch, für die Bevölkerung gleiche Möglichkeiten der Partizipation und Mitwirkung zu schaffen, wenn diese Partizipation mittels

\footnotetext{
1 http://edocket.access.gpo.gov/2009/pdf/E9-1777.pdf.
} 
Informations- und Kommunikationstechnologie erreicht werden soll, denn nicht alle haben denselben Zugang und dieselbe Affinität zu den entsprechenden Technologien (Busch 2010).

\section{Effizienz, Effektivität und Public Value}

Parallel zur Eröffnung neuer Partizipationsmöglichkeiten durch die Digitalisierung wird im Bereich der öffentlichen Verwaltung darüber debattiert, welche Wertekriterien für Aktivitäten im öffentlichen Sektor eigentlich gelten (sollen). Im traditionellen Public Management standen vorwiegend Größen der Kosteneffizienz im Zentrum; dies wurde jedoch von mehreren Autoren kritisiert (bspw. Cordella und Bonina 2012). Durch das unternehmerische Denken im New Public Management erweiterte sich das Spektrum um Messwerte für Effektivität (Bryson et al. 2014). Für den Verwaltungswissenschaftler Moore (1995) spielt aber bei der Bewertung von öffentlichen Arbeiten eine ebenso wichtige Rolle, inwieweit sie soziale Gleichheit schaffen oder die Werte der Demokratie wahren. Im Kern geht es hier also um die Frage, was denn die Aufgabe von Entscheidungsträgern im öffentlichen Sektor ist (Stoker 2006).

Der Public Value bezeichnet den Wert, den die Gesellschaft (bspw. staatlichen) Handlungen beimisst. Da das Wertespektrum von Individuen und der Gesellschaft, die sie bilden, über rein monetäre und effizienzbezogene Aspekte hinausgeht, spiegelt dieses Konstrukt auch ein ganzheitlicheres Wertespektrum wider. Public Value gibt den Wert an, der durch öffentliche Dienstleistungen und Produkte geschaffen wird (Moore 1995). Dieser Wert wird aber nicht aus organisationaler Sicht mittels statistischer Modelle, Kosteneffizienzanalysen oder Kosten-Nutzen-Berechnungen gemessen, sondern von den Individuen selbst angegeben (Meynhardt 2009). Bei diesem Ansatz wechseln also nicht nur die Methoden der Bewertung, sondern auch die Akteure: Während es im New Public Management vorwiegend Experten sind, die öffentliche Aktivitäten bewerten, wird beim Public-Value-Ansatz mittels partizipativer und konsultativer Verfahren die Bevölkerung beigezogen (Moore 1995).

Obwohl der Ansatz des Public Value bereits seit 1995 existiert, gibt es bislang kaum empirische Arbeiten, die Public Value operationalisieren und messen. Vor diesem Hintergrund haben Scott et al. (2015) einen Fragebogen als Bewertungsinstrument entwickelt. Damit bewerten sie in ihrer Studie aber Informationsseiten zu Steuern, Einwohnerdiensten etc., nicht partizipative Prozesse des öffentlichen Sektors (Scott et al. 2015).

Unsere Studie baut auf dieser Arbeit auf: Wir verwenden die Items von Scott et al. (2015), um mithilfe einfacher Fragebogenmethodik den Public Value von OnlinePartizipation zu messen. Dies tun wir exemplarisch anhand eines laufenden OpenGovernment-Projekts. 


\section{Das Fallbeispiel: „Züri wie neu“}

„Züri wie neu“ ist eine Open-Government-Plattform der Stadt Zürich, die die Zusammenarbeit zwischen Verwaltung und Bevölkerung intensivieren und fördern soll (Stürmer und Ritz 2014). Es handelt sich dabei um ein Siegerprojekt des Ideenwettbewerbs zum Legislaturschwerpunkt „eZürich“" , wobei die gleiche Idee bereits in anderen Städten umgesetzt wurde oder mittlerweile umgesetzt wird ${ }^{3}$. Die Idee des Projekts ist, dass die Zürcher Bevölkerung Mängel und Schäden, die sie an der städtischen Infrastruktur feststellt, via Smartphone-App oder Webanwendung ${ }^{4}$ direkt und unkompliziert der Stadt melden kann. Das kann unterschiedlichste Mängel betreffen, beispielsweise Schlaglöcher in Straßen, defekte Lichtinstallationen, Tierkadaver oder Beschädigungen durch Vandalismus. Vom Start des Projekts im April 2013 bis zum Dezember 2016 gingen auf der Internetplattform an die 10.000 Meldungen ein, das entspricht zirka sieben Benachrichtigungen pro Tag; die Tendenz ist steigend. ${ }^{5}$

Eine Meldung bei „Züri wie neu“ abzugeben ist einfach: Entweder man öffnet die Webanwendung www.zueriwieneu.ch, trägt dort die Schadensmeldung inklusive eines Fotos ein, platziert einen Zeiger auf der Stadtkarte, um den Standort anzugeben, und schickt das Formular ab. Oder man startet die iPhone- oder Android-App „Züri wie neu“, fotografiert den betreffenden Mangel, fügt eine kurze Beschreibung hinzu und übermittelt der Stadtverwaltung die Meldung mit einer GPS Angabe des Standorts. In beiden Fällen müssen E-Mail-Adresse und Telefonnummer in das Meldeformular eintragen werden. Die persönlichen Daten werden nur stadtintern für etwaige Rückfragen erfasst, sie werden weder an Dritte weitergegeben noch veröffentlicht. Außerdem muss jeweils die Schadenskategorie (Signalisation, Beleuchtung, Graffiti, Grünflächen etc.) angegeben werden, damit die Meldung möglichst rasch der jeweils zuständigen Behördenstelle zugestellt werden kann.

Wenn die Meldung bei der Züricher Stadtverwaltung eingeht, wird dort zunächst geprüft, ob die inhaltlichen Vorgaben (keine Verbesserungsvorschläge, keine diffamierenden Äußerungen etc.) eingehalten wurden. Dann wird sie innerhalb eines Arbeitstags an die zuständige Stelle weitergeleitet, die sich um die inhaltliche Bearbeitung kümmert. Diese Stelle geht das Problem innerhalb von fünf Arbeitstagen an und gibt dann dem Absender der Meldung ein Feedback ${ }^{6}$. Daneben werden alle Meldungen auch öffentlich zugängig gemacht und auf einer Karte eingetragen (siehe Abb. 1). Ein genaues Datum der Mängelbehebung wird nicht immer angeboten, da die verschiedenen zuständigen Stellen weitgehend autonom in der Formulierung der Antworten und im Streben nach erhöhter Präzision sind. Auch weil die Anforderungen und Umstände in der Mängelbehebung sehr unterschiedlich sind. Beispielsweise müssen Beschädigungen an der Beleuchtung möglichst schnell repariert werden, der Abfall wird auf der regulären Tour entsorgt und Graffitis stehen hingegen oftmals

\footnotetext{
2 https://www.zueriwieneu.ch.

3 http://fixmystreet.org/sites/.

4 https://www.zueriwieneu.ch.

5 Siehe dazu die Datenanalyse unter https://zueriwieneu.fdn.iwi.unibe.ch/.

6 https://www.zueriwieneu.ch/faq.
} 


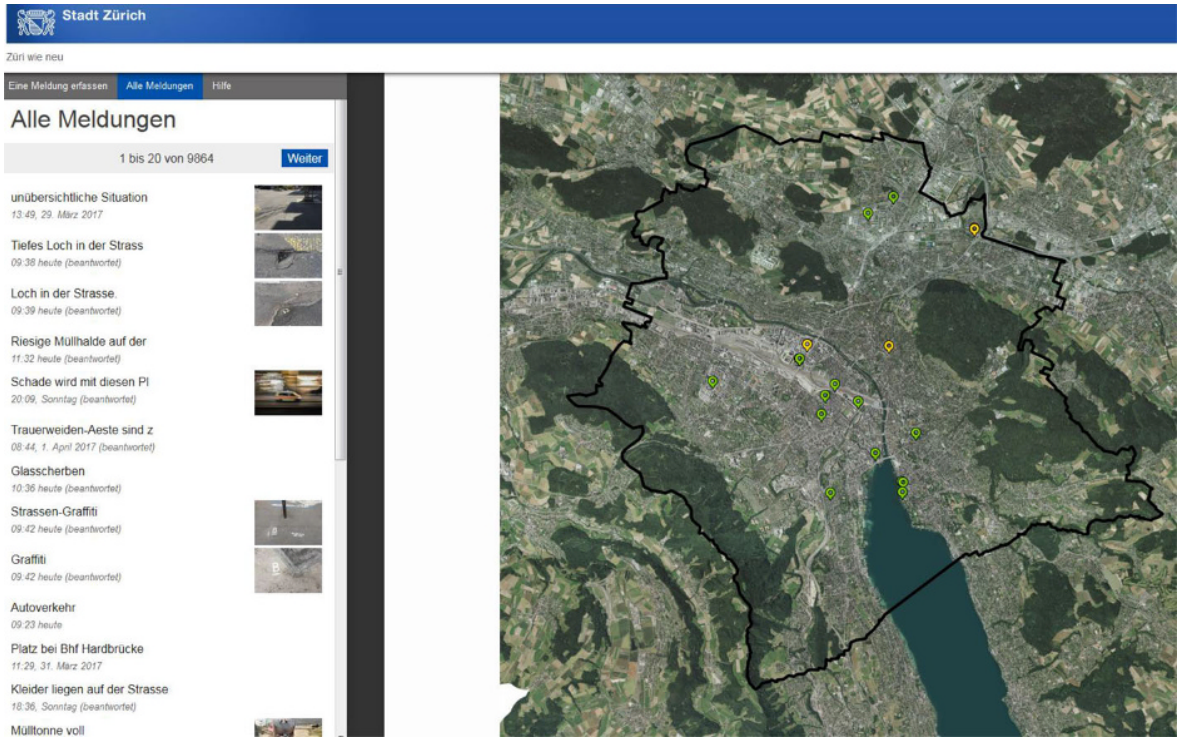

Abb. 1 Auszug aus der Webapplikation

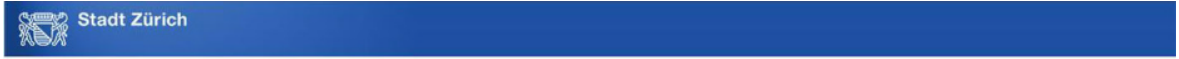

Züri wie neu

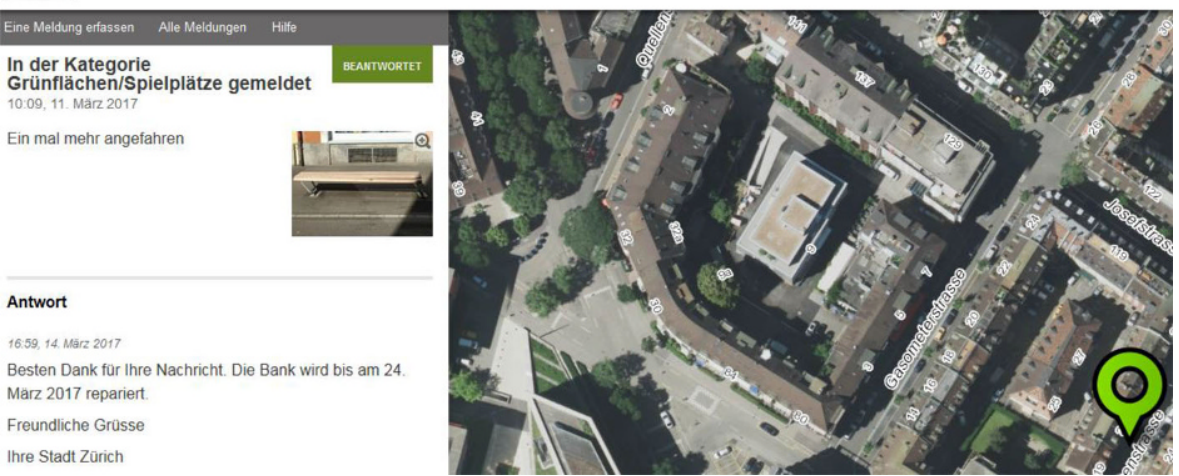

Abb. 2 Antwort der Stadt

auf privatem Grund. In Abb. 2 ist eine beispielhafte Antwort der Stadtverwaltung zu sehen.

Der Nutzen des Projekts ist ein doppelter: Einerseits kann die Bevölkerung auf einfache Art und Weise einen Betrag zur städtischen Infrastruktur leisten. Andererseits erhält die Stadtverwaltung Unterstützung bei der Feststellung von Mängeln ${ }^{7}$.

\footnotetext{
7 https://www.stadt-zuerich.ch/ted/de/index/departement/medien/medienmitteilungen/2013/april/130416a. html.
} 
Nachdem in der Konzeptphase mithilfe von Experteninterviews alle wichtigen Fragen bezüglich Aufwand, Technik und Organisation der Online-Plattform geklärt worden waren, wählte die Stadt Zürich für die Umsetzung einen Partner aus Großbritannien: Die Organisation mySociety ${ }^{8}$, die bereits erfolgreich eine eigene OpenSource-Plattform namens FixMyStreet ${ }^{9}$ realisiert hatte, erfüllte mit ihrer Lösung alle Anforderungen der Stadt Zürich (Gees 2013; Habenstein et al. 2016). Aufgrund der positiven Rückmeldungen der Bevölkerung und der Medien entschied der Stadtrat nach Abschluss der Pilotphase im Jahr 2014, das Projekt in den Regelbetrieb zu überführen. Insgesamt erwies sich das eingeführte Mängelmeldeportals als großer Erfolg, denn es hat nicht nur die Zusammenarbeit zwischen der Stadtverwaltung und der Bevölkerung intensiviert, sondern leistet auch einen wichtigen Beitrag zu einer intakten Infrastruktur und einer sauberen Stadt.

\section{Ergebnisse der Umfrage}

An 2613 Benutzer von „Züri wie neu“ wurde ein Online-Fragebogen per Email versendet. Dabei wurden Fragen zu der Qualität, dem Nutzen, der Motivation der Teilnehmenden und demografische Merkmale gestellt. Bis zum Stichtag im Sommer 2016 gingen 738 vollständig ausgefüllte Fragebögen ein. Die für den Public Value relevanten Fragen sind nachfolgend aufgelistet, wobei die Fragen in die Konstrukte Zeitgewinn, Komfort, Kommunikation, Informiertheit, Partizipation und Vertrauen unterteilt sind. Die Items sind in Anlehnung an Scott et al. (2015) formuliert worden (Tab. 1).

Die Abb. 3, 4 und 5 im Anhang veranschaulichen die Resultate der Umfrage: Der rote Bereich links zeigt jeweils die Antworten, die eine geringe Übereinstimmung mit dem betreffenden Item angeben, der grüne Bereich rechts repräsentiert eine hohe Übereinstimmung mit dem Item. Insgesamt lässt bereits das überdurchschnittliche Vorhandenseins von Zustimmung darauf schliessen, dass die Teilnehmenden die Anwendung als wertvoll einstufen.

Die Auswertung ergab überdurchschnittlich hohe Zustimmungswerte bei Zeitgewinn (Effizienz), Komfort und Kommunikation (beides Effektivität), aber auch bei der Bildung von Vertrauen in die Verwaltung, der wahrgenommenen eigenen Informiertheit und dem Gefühl, an wichtigen Entscheidungen zu partizipieren (alles soziale Werte). Unsere Umfrage bietet somit einen weiteren Beleg, dass OnlinePartizipation Public Value kreiert.

Dem Public-Value-Ansatz entspricht besonders das Ergebnis, dass die Befragten Online-Partizipation als etwas ansehen, was der Bevölkerung zugutekommt. Sie nehmen diese Form der Beteiligung also nicht so wahr, dass der Staat die Bürger als Informationsgeber missbraucht, sondern sie betrachten sie als Dienstleistung für die Bevölkerung. Dies wird auch dadurch unterstrichen, dass ,Züri wie neu“ nach Ansicht der meisten Befragten seinen Zweck tatsächlich erfüllt.

\footnotetext{
8 https://www.mysociety.org.

9 https://fixmystreet.org.
} 
Tab. 1 Items zur Evaluation von Public Value

Zeitgewinn

„Züri wie neu“ spart mir Zeit

Mit „Züri wie neu“ erhalte ich schneller Antwort als über herkömmliche Meldeverfahren

Mit „Züri wie neu“ bekomme ich Dinge schneller geregelt

Dank „Züri wie neu“ kann ich den direkten Kontakt mit der Verwaltung vermeiden

Komfort

Es ist mir wichtig, dass die Benutzung von „Züri wie neu“ jederzeit möglich ist

Es ist mir wichtig, dass der Zugriff auf ,Züri wie neu“ von überall möglich ist

„Züri wie neu“ ist so flexibel, dass ich nicht von anderen Tätigkeiten abgehalten werde Kommunikation

„Züri wie $\quad$ Ermöglicht eine effiziente Kommunikation mit der Stadt Zürich

neu“"

Informiertheit

„Züri wie Verbessert mein Verständnis für die Dienstleistungen der Stadt Zürich

neu“ Erhöht mein Wissen zu Themen, die mir wichtig sind

Erlaubt mir, auf verschiedene Bedürfnisse Antworten zu erhalten

Partizipation

„Züri wie $\quad$ Erlaubt mir, Einfluss zu nehmen auf Dinge, die mir wichtig sind

neu“

Verstärkt mein Gefühl, Teil einer aktiven Demokratie zu sein

Gibt mir das Gefühl, dass Entscheidungsträger mich anhören

Gibt mir das Gefühl, dass ich bei wichtigen Angelegenheiten gefragt werde

Vertrauen

„Züri wie neu“ dient dem Interesse der Bevölkerung

„Züri wie neu“ benutze ich gerne, weil meine Anfragen effizient bearbeitet werden

Ich kann mich immer auf „Züri wie neu“ verlassen

Ich verlasse mich darauf, dass ,Züri wie neu“ seinen Zweck erfüllt

\section{Diskussion: Implikationen und Limitationen der Ergebnisse}

In der Einleitung haben wir die Frage gestellt, ob Online-Partizipation im öffentlichen Sektor von der Bevölkerung als wertvoll empfunden wird. Dies können wir anhand der theoretischen Erarbeitung und der empirischen Resultate der Umfrage klar bestätigen. Dass die BürgerInnen dabei die Faktoren für Effektivität und Effizienz sehr positiv bewerten, ist nicht überraschend. Es könnte vor allem daran liegen, dass die Bevölkerung sich aufgrund des föderalistischen Systems der Schweiz oft nicht darüber im Klaren ist, an wen sie sich mit welchem Problem wenden muss. Dann steigt eine zentrale Anlaufstelle aus ihrer Sicht die Effizienz und Effektivität erheblich.

Aus der Sicht des New Public Management könnte allerdings gefragt werden, was den Mehraufwand und die Kosten einer solchen Applikation legitimiert; schließlich kann es der öffentlichen Hand gleichgültig sein, wie effizient und effektiv die Bevölkerung infrastrukturelle Mängel melden kann. Zudem erscheint es aus dieser Perspektive kaum sinnvoll, Online-Partizipationstools für diesen Zweck zu imple- 
mentieren, weil die von der Stadt Zürich bereitgestellte Infrastruktur bereits auf einem sehr hohen Niveau ist. Gerade in dieser Hinsicht ist der Public Value hilfreich: Eine ganzheitliche Evaluation dieser Maßnahme zeigt sehr deutlich, dass die Bevölkerung Online-Partizipation als wertvoll empfindet, und sie gibt Aufschluss darüber, dass ihr Nutzen vor allem darin liegt, das Vertrauen in die Stadt Zürich zu erhöhen. Vertrauen in die öffentliche Verwaltung ist insofern sehr wichtig, als andernfalls in Abstimmungen Budgets nicht bewilligt, neue Initiativen zur Kontrolle staatlicher Aktivitäten gestartet oder Personen abgewählt werden könnten (Bryson 2004). Die einzige Legitimation der öffentlichen Hand, Geld auszugeben, liegt darin, den Bedürfnissen der Bevölkerung nachzukommen (Moore 1995).

Unsere Studie hat jedoch auch gewisse Limitationen: Erstens hat die Stadt Zürich, wie schon erwähnt, bereits einen sehr hohen Standard in der Infrastruktur und verfügt über sehr detailliertes Wissen über den Zustand der Infrastruktur in der Stadt. Es ist fraglich, ob der Public Value dieser Massnahme ebenso hoch wäre, wenn sie in einer Stadt eingeführt würde, wo die Beantwortungszeit viel schlechter wäre. Zweitens bezieht sich die Maßnahme auf die Partizipation im öffentlichen Sektor in Bezug auf Schadensmeldungen, nicht Verbesserungsvorschläge. Daher erlauben die Ergebnisse keine Rückschlüsse auf Online-Partizipation in der Privatwirtschaft (bspw. Open Innovation). Drittens ist für die Schweiz ein hohes Vertrauen in die Regierung kennzeichnend. Es ist fraglich, ob der Public Value einer solchen Maßnahme in Ländern, wo die Bevölkerung weniger Vertrauen in die Regierung setzt, ähnlich hoch ausfallen würde. Viertens schließlich geht es bei Public Value nicht nur um das Wohl derjenigen, die den betreffenden Service genutzt haben, sondern um das Gemeinwohl, und das umfasst auch die BürgerInnen, die nicht über diesen Service partizipiert haben. Deshalb müssten für eine noch umfassendere Werterhebung auch Leute befragt werden, die nicht Gebrauch gemacht haben vom Mängelmeldeportal.

Und dennoch, „Züri wie neu“ zeigt auf eindrückliche Weise, dass soziale Vorteile von solchen Open Government Plattformen wichtig sind für die Bevölkerung. So kann davon ausgegangen werden, dass solche Mängelmelder und auch weitere Partizipations-Anwendungen in Zukunft immer öfter angetroffen werden. 


\section{Anhang}

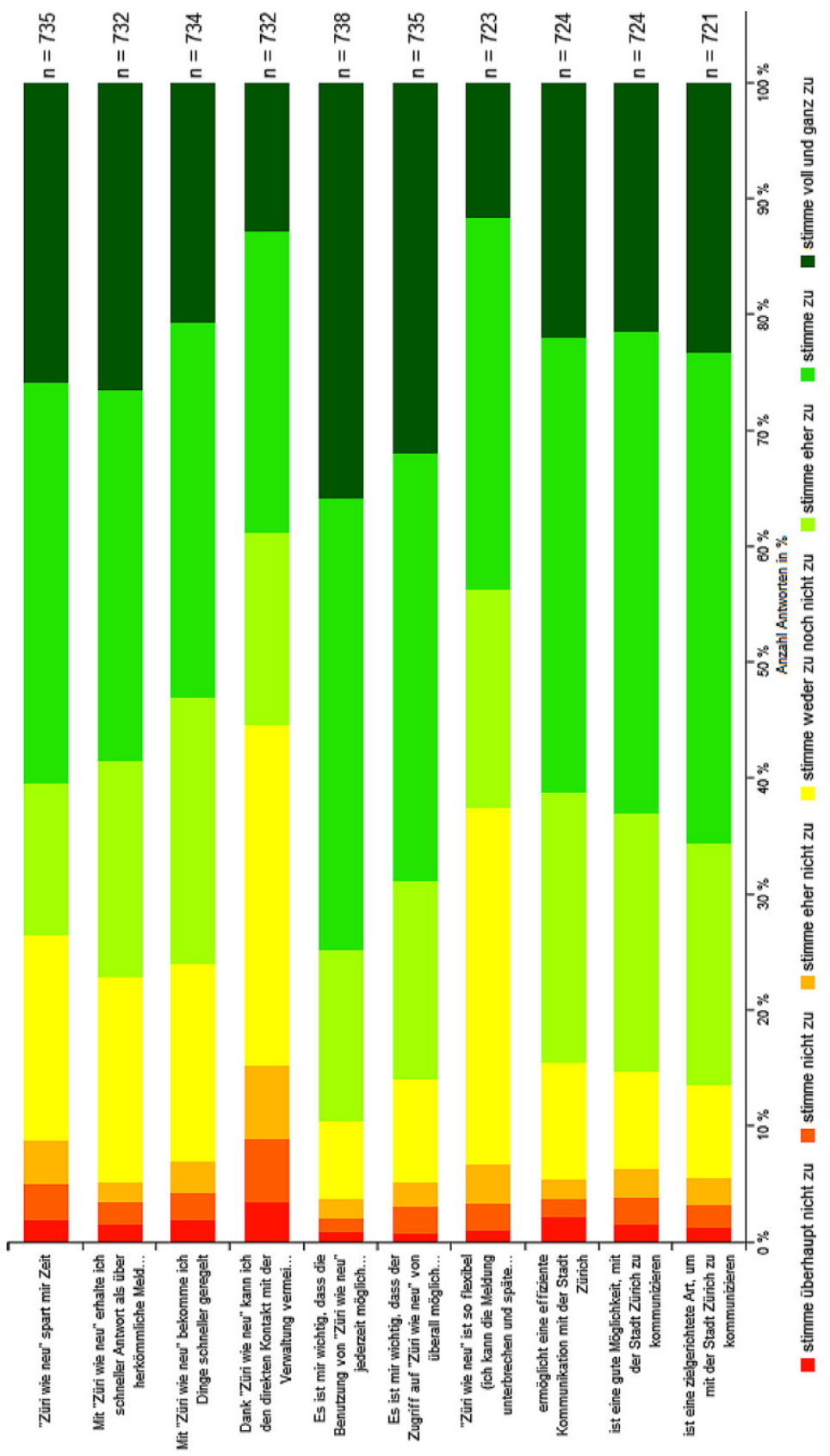

Abb. 3 Effizienz und Nutzen aus der Benutzerperspektive (Die grafische Visualisierung der Antworten (Abb. 3, 4 und 5) wurde von der Forschungsstelle Digitale Nachhaltigkeit erstellt (https://zueriwieneu.fdn. iwi.unibe.ch/) 


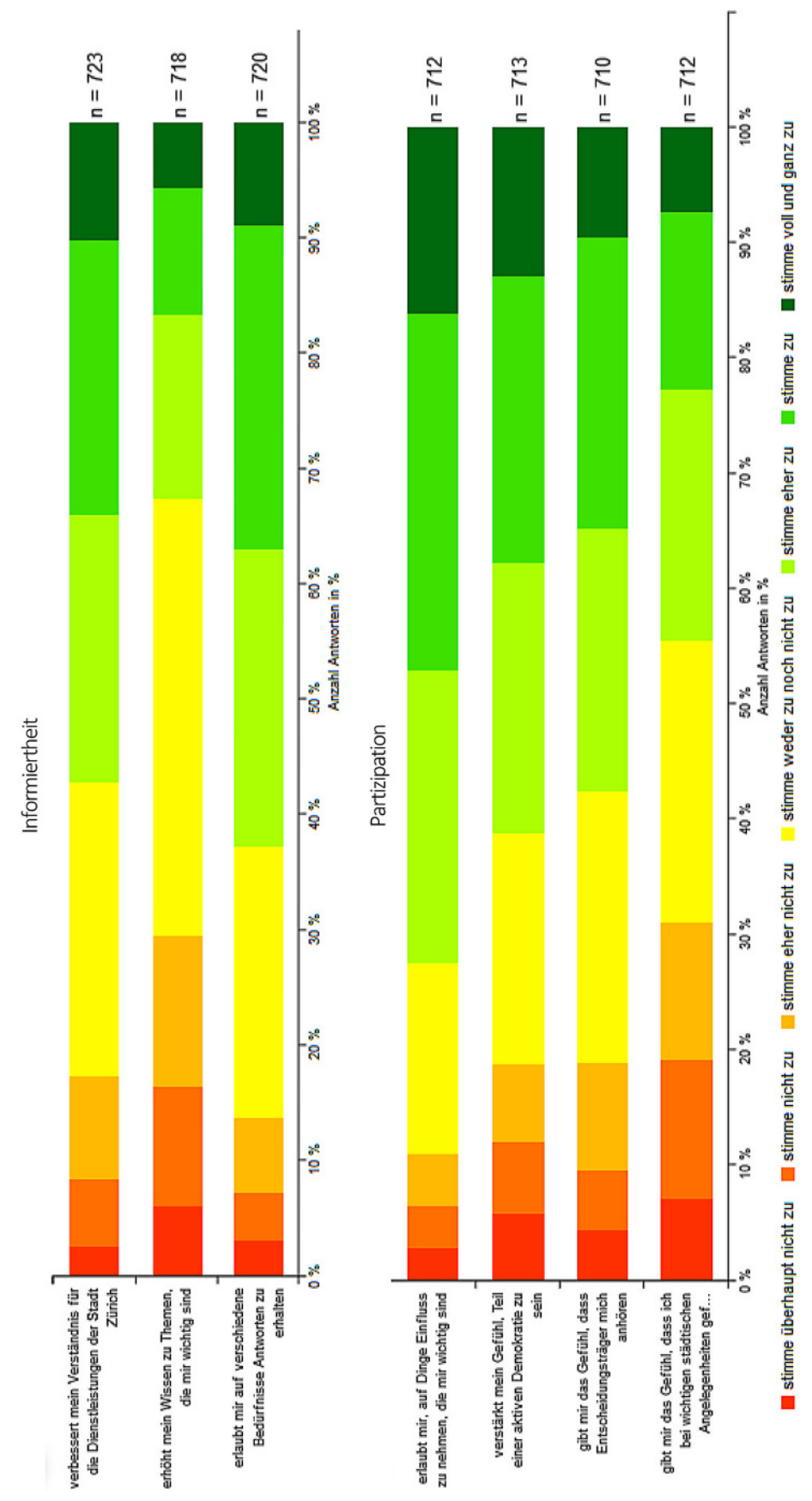

Abb. 4 Informiertheit und Partizipation 
Abb. 5 Vertrauen in die Verwaltung

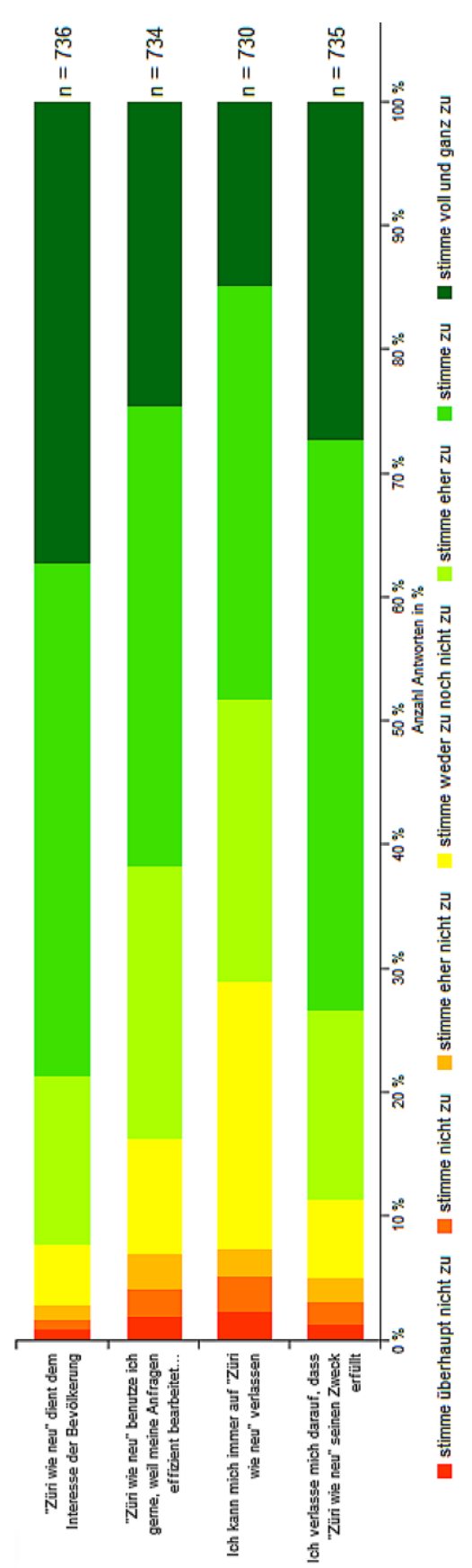


Open Access Dieser Artikel wird unter der Creative Commons Namensnennung 4.0 International Lizenz (http://creativecommons.org/licenses/by/4.0/deed.de) veröffentlicht, welche die Nutzung, Vervielfältigung, Bearbeitung, Verbreitung und Wiedergabe in jeglichem Medium und Format erlaubt, sofern Sie den/die ursprünglichen Autor(en) und die Quelle ordnungsgemäß nennen, einen Link zur Creative Commons Lizenz beifügen und angeben, ob Änderungen vorgenommen wurden.

\section{Literatur}

Bryson JM (2004) What to do when Stakeholders matter: stakeholder identification and analysis techniques. Public Manag Rev 6:21-53. doi:10.1080/14719030410001675722

Bryson JM, Crosby BC, Bloomberg L (2014) Public value governance: moving beyond traditional public administration and the new public management. Public Adm Rev 74:445-456

Busch T (2010) Capabilities in, capabilities out: overcoming digital divides by promoting corporate citizenship and fair ICT. Ethics Inf Technol 13:339-353. doi:10.1007/s10676-010-9261-3

Collins S (2009) Government 2.0, e-Government and culture. Government 2:79-97

Cordella A, Bonina CM (2012) A public value perspective for ICT enabled public sector reforms: a theoretical reflection. Gov Inf Q 29:512-520

Denhardt RB, Denhardt JV (2000) The new public service: serving rather than steering. Public Adm Rev 60:549-559. doi:10.1111/0033-3352.00117

Dunleavy P, Hood C (1994) From old public administration to new public management. Public Money Manag 14:9-16

Fiorino DJ (1990) Citizen participation and environmental risk: a survey of institutional mechanisms. Sci Technol Hum Values 15:226-243

Gees C (2013) Züri wie neu: Siegerprojekt aus eZürich-Ideenwettbewerb ist online. Geomatik Schweiz 111(6). doi: $10.5169 /$ seals-323423

Habenstein A, D’Onofrio S, Portmann E, Stürmer M, Myrach T (2016) Open Smart City: Good Governance für smarte Städte. Smart City:47-71. doi:10.1007/978-3-658-15617-6_3

Hilgers D (2012) Open government: theoretische Bezüge und konzeptionelle Grundlagen einer neuen Entwicklung in Staat und öffentlichen Verwaltungen. Z Betriebswirtsch 82:631-660

Meynhardt T (2009) Public value inside: what is public value creation? Int J Public Adm 32:192-219. doi: $10.1080 / 01900690902732632$

Moore MH (1995) Creating public value: strategic management in government. Harvard university press, Cambridge

Obama B (2009) Transparency and open government. Memorandum for the heads of executive departments and agencies. http://edocket.access.gpo.gov/2009/pdf/E9-1777.pdf. Zugegriffen: 22.12.2016

Osborne DE, Gaebler T (1992) Reinventing government: how the entrepreneurial government is transforming the public sector. Plume, New York

Scott M, DeLone W, Golden W (2015) Measuring eGovernment success: a public value approach. Eur J Inf Syst. doi:10.1057/ejis.2015.11

Stoker G (2006) Public value management: a new narrative for networked governance? Am Rev Public Adm 36:41-57

Stürmer M, Ritz A (2014) Public Governance durch Open Government: Zwei sich ergänzende Ansätze für die staatliche Aufgabenerfüllung der Zukunft. Jahrb Schweiz Verwaltungswiss 2014:125-138

Walter-Busch E (1996) Organisationstheorien von WEBER bis WEICK. G+B Verlag Fakultas, Amsterdam Weber M (1922) Wirtschaft und Gesellschaft, Grundriss der verstehenden Soziologie. J. CB Mohr, Tübingen 